\title{
Subxiphoid approach for spontaneous bilateral pneumothorax: a case report
}

\author{
Matthew Fok ${ }^{1}$, Jay Karunanantham ${ }^{1}$, Jason M. Ali ${ }^{1}$, Serena Concina ${ }^{2}$, Shruti Jayakumar ${ }^{2}$, Adam Peryt ${ }^{1}$, \\ Aman Coonar', Giuseppe Aresu' \\ ${ }^{1}$ Department of Thoracic Surgery, Papworth Hospital NHS Foundation Trust, Cambridge, UK; ${ }^{2}$ Department of General Surgery, Santa Maria della \\ Misericordia University Hospital, Udine, Italy \\ Correspondence to: Mr Giuseppe Aresu, MD, PhD. Department of Thoracic Surgery, Papworth Hospital NHS Foundation Trust, Papworth Everard, \\ Cambridge, CB23 3RE, UK. Email: giuseppe.aresu@nhs.net.
}

\begin{abstract}
The development of video-assisted thoracoscopic surgery (VATS) has contributed to reduced pain and improved recovery following thoracic surgery. However, pain remains a major issue. Patients with bilateral pulmonary pathology requiring operative intervention may have even more pain due to bilateral transthoracic incisions. The recently described uniportal subxiphoid VATS approach provides an opportunity to undertake bilateral thoracic surgery through a single incision that avoids the bilateral intercostal nerve damage caused by transthoracic incision and drainage. Here we report a case of a patient requiring bilateral bullectomy and pleurectomy for the management of pneumothorax that was performed successfully by the subxiphoid VATS approach.
\end{abstract}

Keywords: Subxiphoid; video-assisted thoracoscopic surgery (VATS); bilateral pneumothorax; uniportal

Received: 18 September 2017; Accepted: 10 October 2017; Published: 27 October 2017.

doi: $10.21037 /$ jovs.2017.10.02

View this article at: http://dx.doi.org/10.21037/jovs.2017.10.02

\section{Introduction}

Primary spontaneous pneumothorax (PSP) is a common emergency room presentation typically occurring in young, low body weight males, who are otherwise healthy without clinically apparent lung disease in their late teens or third decade of life. Bilateral PSP is a rare condition, accounting for $\sim 1.3 \%$ of spontaneous pneumothoraces (1). Surgical resection of the blebs or bullae typically found in these patients, offers good long-term outcomes significantly decreasing the risk of recurrence (2).

At present the most common surgical practice in patients presenting with bilateral pneumothoraces is with multiport bilateral video-assisted thoracoscopic surgery (VATS). This technique has evolved to the use of bilateral uniportal VATS requiring one incision on either side to perform pleurectomy and bullectomy $(3,4)$. Recently, to further minimise the surgical impact, a select number of centres has adopted the uniportal subxiphoid VATS (SVATS) technique which could offer several advantages including reducing acute and chronic postoperative pain by avoiding damage to the intercostal nerves, with consequential benefit in terms of recovery and quality of life (5-9). The ability to access both pleural cavities using a single incision is a further advantage of uniportal subxiphoid VATS which can be utilized in the management of patients with can bilateral pneumothoraces. This case report describes our procedure and technique for performing uniportal subxiphoid bilateral bullectomy and pleurectomy.

\section{Case presentation}

An 18-year-old man presented to the Emergency Department (ED) with sudden onset chest pain and a nonproductive cough. He had no significant past medical history or underlying pulmonary disease and there was no history of trauma preceding the onset of symptoms. On admission the patient was dyspnoeic and had decreased breath sounds over the right hemithorax. He was haemodynamically stable with oxygen saturation of $94 \%$ on room air. Complete blood count and biochemistry results were within normal 


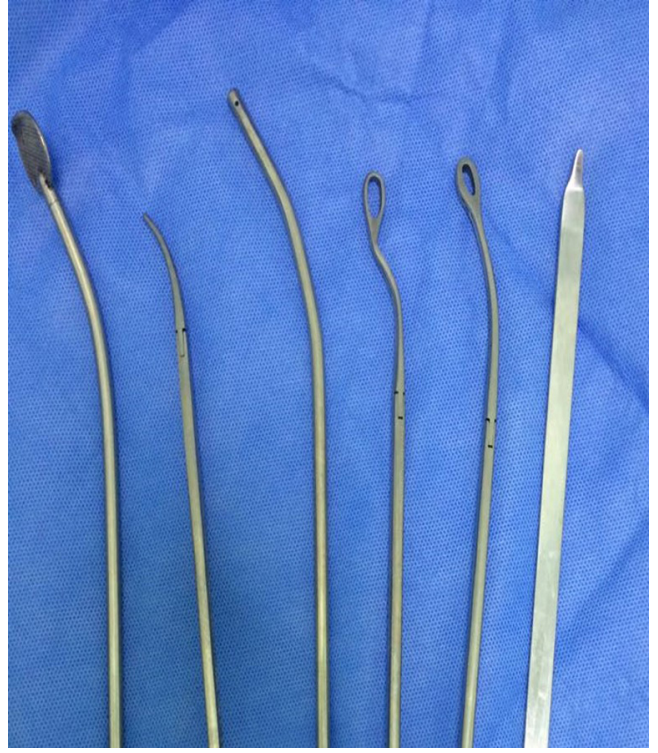

Figure 1 Subxiphoid VATS instrument set [Shanghai Medical Instruments (Group) Ltd]. VATS, video-assisted thoracoscopic surgery.

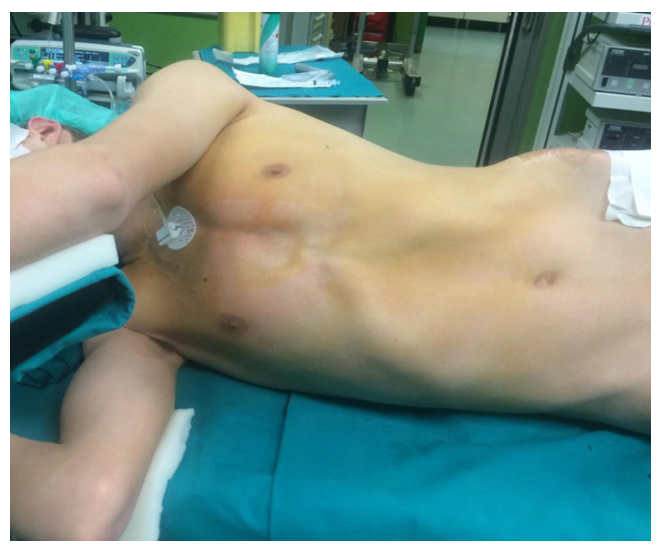

Figure 2 Positioning of patient for left sided subxiphoid approach VATS surgery. VATS, video-assisted thoracoscopic surgery.

limits. A chest radiograph demonstrated a large right sided pneumothorax which was treated with insertion of a $14 \mathrm{Fr}$ Seldinger chest drain and resolved spontaneously during a 2-day admission and he was discharged home. One month later he presented to the ED with similar symptoms and at this time chest radiography demonstrated a left sided pneumothorax which was treated with insertion of a 14 Fr Seldinger chest drain but had a persistent air leak. Chest computed-tomography (CT) revealed multiple subcentimeter blebs of variable size in the apical portion of both lungs. He was referred to our tertiary cardiothoracic centre for consideration of surgical treatment.

\section{Patient selection and workup}

Indications for thoracoscopic pleurectomy and bullectomy include patients following a second ipsilateral spontaneous pneumothorax or a first contralateral pneumothorax. Other patients who warrant consideration for surgical management include patients with spontaneous pneumothorax with: prolonged air leak, incomplete lung expansion, bilateral pneumothorax, tension pneumothorax or blebs on CT imaging (10). Contraindications to a subxiphoid approach include high BMI, patients with cardiomegaly and reoperative procedures.

\section{Instruments}

Standard VATS instruments with dedicated instruments specially designed for SVATS [Shanghai Medical Instruments (Group) Ltd., Shanghai, China] are used during the operations (Figure 1).

\section{Surgical procedure}

A single staged bilateral surgical intervention was planned. The operation was performed with the patient under general anaesthesia and double lumen endotracheal tube intubation to allow for selective lung ventilation. Patient was placed in a left lateral decubitus position slightly rotated posteriorly in order to optimize exposure of the subxiphoid region for the left-sided procedure (Figure 2). The operating surgeon is positioned in front of the patient and the assistant stands more caudally on the side being operated on to provide the optimal endoscopic view. Once the left side was completed the patient was positioned similarly, to the right, in order to access the right pleural cavity.

A $3 \mathrm{~cm}$ midline vertical incision was made below the sternocostal triangle to expose the xiphoid process. Dissection of the linea alba was performed and the pleural cavity was entered by blunt finger dissection above the level of the diaphragm. An Alexis soft tissue wound retractor (Applied Medical CA, USA) was positioned to create a space for instrument and a 30 degree $10 \mathrm{~mm}$ camera insertion (Karl Storz, Tuttlingen, Germany).

The left lung was excluded from ventilation and the pleural inspected. All instruments to complete the 


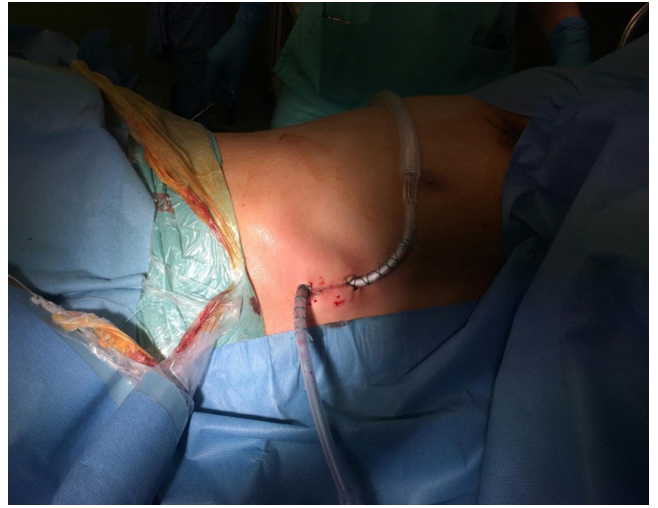

Figure 3 Immediate post-operative appearance with drains to each pleural space.

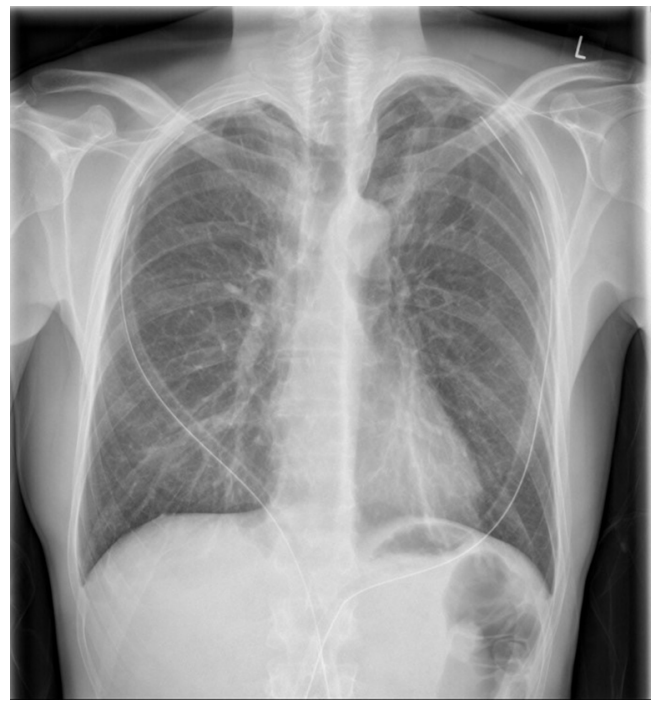

Figure 4 Chest radiograph demonstrating drain position postoperatively.

procedure were placed through the single port, cranially to the camera. An experienced assistant is required to provide the operator with the appropriate views at all times and to avoid instrument clashes.

Blebs were identified at the apex of both the left and right upper lobes and were resected using a surgical stapler (Medtronic EndoGIA, MA, USA). Apical and mid-zone pleurectomy was performed bilaterally with pleural abrasion to the lower zones. Two $28 \mathrm{Fr}$ drains were placed via the subxiphoid incision (Figures 3 and 4 ) and placed on $-2 \mathrm{KPa}$ suction using a Thopaz system (Medela, Switzerland). The patient was mobilized from day 0 with minimal pain. The drains were removed once the air leak had ceased on the $4^{\text {th }}$

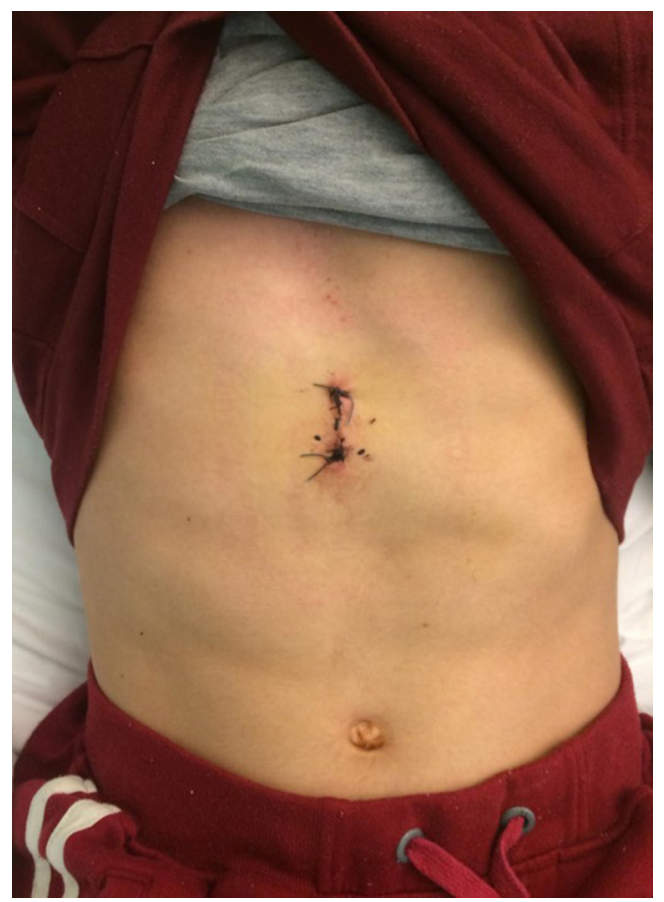

Figure 5 Appearances of surgical wound following drain removal.

postoperative day (Figure 5).

\section{Discussion}

VATS is associated with decreased postoperative pain, reduced length of hospital stay and fewer postoperative complications compared to open thoracotomy (11). VATS is now regarded by many surgeons as the gold standard in the surgical management of a variety of thoracic conditions such as spontaneous pneumothorax, pleural disease and for lung resections. In 2004 Rocco et al. described the uniportal VATS technique for the treatment of PSP with the potential advantage of reducing postoperative pain and faster recovery (3). Nonetheless even small chest wall incision cause intercostal neuralgia and can result in chronic thoracic pain. The subxiphoid approach is a novel technique which is only performed in a select number of centres worldwide but its use has been described in a variety of cardiothoracic operations including pericardial window creation, coronary artery bypass surgery, thymectomy, lobectomy and pulmonary wedge resections $(5-9,12)$.

Since the chest drains are not entering the thoracic cavity through intercostal spaces, they do not impinge on the intercostal neurovascular bundle during mobilization of the patient. This has the potential benefit of reducing post- 
operative pain and allows for early aggressive mobilization and clearance of secretions with the benefit of reducing the incidence of venous thromboembolism and lower respiratory tract infection (6). Earlier mobilization may also facilitate reduced length of hospital stay.

The subxiphoid VATS technique can be easily learnt by surgeons experienced in uniportal VATS. The camera views and instrument positioning can be more challenging than for conventional VATS but the necessary skills can be acquired over a short learning curve, particularly for minor procedures.

\section{Acknowledgements}

None.

\section{Footnote}

Conflicts of Interest: The authors have no conflicts of interest to declare.

Informed Consent: Written informed consent was obtained from the patient for publication of this manuscript and any accompanying images.

\section{References}

1. Graf-Deuel E, Knoblauch A. Simultaneous bilateral spontaneous pneumothorax. Chest 1994;105:1142-6.

2. Ben-Nun A, Soudack M, Best LA. Video-assisted thoracoscopic surgery for recurrent spontaneous pneumothorax: the long-term benefit. World J Surg

doi: 10.21037 /jovs.2017.10.02

Cite this article as: Fok M, Karunanantham J, Ali JM, Concina S, Jayakumar S, Peryt A, Coonar A, Aresu G. Subxiphoid approach for spontaneous bilateral pneumothorax: a case report. J Vis Surg 2017;3:146.
2006;30:285-90.

3. Rocco G, Martin-Ucar A, Passera E. Uniportal VATS wedge pulmonary resections. Ann Thorac Surg 2004;77:726-8.

4. Kim KS. Single-staged uniportal VATS in the supine position for simultaneous bilateral primary spontaneous pneumothorax. J Cardiothorac Surg 2017;12:25.

5. Aresu G, Jiang L, Bertolaccini L. Subxiphoid videoassisted major lung resections: the Believers' speech. J Thorac Dis 2017;9:E387-E389.

6. Hernandez-Arenas LA, Lin L, Yang Y, et al. Initial experience in uniportal subxiphoid video-assisted thoracoscopic surgery for major lung resections. Eur J Cardiothorac Surg 2016;50:1060-6.

7. Hernandez-Arenas LA, Guido W, Jiang L. Learning curve and subxiphoid lung resections most common technical issues. J Vis Surg 2016;2:117.

8. Aresu G, Wu L, Lin L, et al. The Shanghai Pulmonary Hospital subxiphoid approach for lobectomies. J Vis Surg 2016;2:135.

9. Aresu G, Weaver H, Wu L, et al. The Shanghai Pulmonary Hospital uniportal subxiphoid approach for lung segmentectomies. J Vis Surg 2016;2:172.

10. MacDuff A, Arnold A, Harvey J, et al. Management of spontaneous pneumothorax: British Thoracic Society Pleural Disease Guideline 2010. Thorax 2010;65 Suppl 2:ii18-31.

11. Reddy RV. The advantages of VATS: a systematic review. Thorax 2005;60:238.

12. Tu CC, Hsu PK. Global development and current evidence of uniportal thoracoscopic surgery. J Thorac Dis 2016;8:S308-18. 